\title{
COMPARISON OF THE SPE MODEL WITH PROTON AND HEAVY ION DATA
}

\author{
Timothy F. Cleghorn and Gautam D. Badhwar \\ NASA Johnson Space Center \\ Houston, Texas, 77058-3696
}

\begin{abstract}
Proton data from the GOES 6 and 7 satellites and heavy ion data from the IMP-8 satellite have been compared to the expected results of Nymmik's new model for solar particle event fluences. This model calculates the energy spectra of ions for protons through nickel for solar particle events, based upon the observed proton integral fluence above $30 \mathrm{MeV}$. Based upon 27 observed proton events of solar cycle 22, and three large historical events, with integral fluences above $30 \mathrm{MeV}$ of greater than $10^{6}$ particles $/ \mathrm{cm}^{2}$, a reasonable agreement with model predictions is seen for more than half of the events. However, several events show a marked departure from the model predictions, leading to the conclusion that there may exist more than a single class of event, or that it may be necessary to include additional parameters within the model, such as solar disk position of the source flare, or height of disturbance in the solar corona. Data for heavy ions, (oxygen and iron), were limited to a total of six solar particle events, of which only two occurred in solar cycle 22 . The agreement between data and the model predictions appeared to be quite good, however this agreement was sensitively dependent upon the value taken for the proton fluence above $30 \mathrm{MeV}$.
\end{abstract}

Introduction

The Model

During the past few years, a model to describe solar particle events has been developed by Nymmik at Moscow State University, (Nymmik, 1997). This model includes descriptions of the energy and/or rigidity spectra of both protons and heavy ions up to nickel. In contrast with earlier work, which suggested an exponential distribution, this model proposes a power law in rigidity above $30 \mathrm{MeV} / \mathrm{nucleon}$, and a flattening of the spectra below that energy. Apart from the fact that a number of natural phenomena can be described by power laws as a result of chaotic or non-linear dynamic behavior, there is no physics per se upon which this model is based. The values for coefficients and exponents are based upon observations of solar particle events, and are therefore statistical in nature.

Solar particle events are generally described and classified by the size, or event integrated fluence above some energy, depending upon the characteristics of the detector(s), and/or the objectives of the researchers human rated spacecraft currently provide shielding against particles with energies below about $30 \mathrm{MeV}$, so it is events with significant numbers of particles above this energy which are of primary importance for this study. For solar particle events, this event integrated fluence $F$ is given by:

$$
F(>30 \mathrm{MeV})=\int_{30}^{\infty} \Phi(E) d E
$$

where $\mathrm{E}$ is the kinetic energy, and $\Phi(\mathrm{E})$ is the differential fluence in protons $/ \mathrm{cm}^{2}-\mathrm{MeV}$. In the proposed model, $\Phi(\mathrm{E})$ is given by:

$$
\Phi(E) d E=C^{*}\left(p / p_{30}\right)^{-\gamma} * d E / \beta
$$


where $\mathrm{p}$ is the momentum, $\mathrm{p}_{30}=239.15 \mathrm{MV}$ is the momentum, (rigidity, $\mathrm{p} / \mathrm{Z}$ ), corresponding to a proton energy of $30 \mathrm{MeV}$. For kinetic energies above $30 \mathrm{MeV}$, the spectral index $\gamma$ is calculated in the model as a function of event size:

$$
\gamma=\left\{\begin{array}{lc}
6.1 & \mathrm{~F}(>30)<10^{6} \\
5.3-0.8 * \sin [\pi / 2 *\{(\log (\mathrm{F}(>30))-7)\}] & 10^{6} \leq \mathrm{F}(>30)<10^{8} \\
4.5 & \mathrm{~F}(>30) \geq 10^{8}
\end{array}\right.
$$

For energies below $30 \mathrm{MeV}$, the spectra flatten, again as a function of the event size. The spectral "droop" index $\alpha$ for the lower energies is given by:

$$
\alpha=0.2 * Y p *(\gamma / 4.5)^{0.75}
$$

where $\mathrm{Yp}=1.0$ for $\mathrm{F}(>30) \geq 3.0 * 10^{7}$

and $\mathrm{Yp}=\mathrm{F}(>30) /(3.0 * 10)^{7}$ for $\mathrm{F}(>30)<3.0 * 10^{7}$

The coefficient $\mathrm{C}$ is given by:

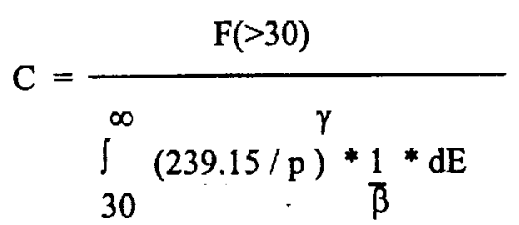

Similarly, the heavy ion spectra are described by power laws in rigidity. The spectral index $\gamma^{(2)}$ is given by:

$$
\gamma^{(z)}=\bar{K} * \gamma^{(p)}
$$

where $\gamma^{(p)}$ is the index for the protons, and $\mathrm{K}$ is a random value of a log normal distribution with mean:

$$
\overline{\log _{10} K}=0.1 \text { or } \overline{\mathrm{K}}=1.26+1-0.07
$$

The coefficients $C^{(z)}=C_{p}^{(z)} * C^{(p)}$ are calculated from tabulated values of $C_{p}^{(z)}$. As with the proton spectra, there exists a "droop" index which describes the shape of the heavy ion spectra below 30 $\mathrm{MeV} /$ nucleon. This index is given by:

$$
\alpha^{(z)}=\alpha^{(p)} *{\frac{\gamma^{(z)}}{\gamma^{(p)}} *(A / Q)}^{-0.47}
$$


where (A/Q) is the mass-to-charge ratio, and is also tabulated in the literature, (Nymmik, 1997). The above expressions are for values of $Z$ between 2 and 28 , although as will be seen below, the solar event data for most heavy ion species are extremely limited.

\section{The Data}

The last solar cycle (solar cycle 22) has provided researchers with almost complete satellite coverage of solar particle events with fluences above $10^{6}$ particles $/ \mathrm{cm}^{2}$ and particle energies above $\sim 1 \mathrm{MeV} /$ nucleon. Approximately 27 solar proton events in this solar cycle have been recognized, although there is some doubt about the duration of some of these, and whether they represent single or multiple events. The integral energy spectra above $1 \mathrm{MeV}$ for 21 of these events has been provided to us kindly by Dr. Herbert Sauer of NOAA, (Sauer, 1997). Six addition events are taken from earlier published literature, (Sauer, 1993; Tylka, et al, 1996). The 21 events provided by Dr. Sauer have integral fluences for energies above: 1, $5,10,30,50,60$, and $100 \mathrm{MeV}$ as does one event from Tylka, et al. In addition, Sauer, 1993 provides integral fluences above 355 and $685 \mathrm{MeV}$ for eight of the 21 , as well as for five other events. It is noted that the refinement of data between the 1993 Sauer publication and the recent set of 21 events does not lead to significant differences, and hence the more recent analyses are used for energies $\leq 100 \mathrm{MeV}$. The solar particle events used in the analyses are listed in Table 1, which also includes the positions of the corresponding solar flare if available.

Data for heavy ions has been taken from Tylka, et al, 1997. This includes spectra for two events in solar cycle 22. These data were obtained using the IMP-8, VLET, and Galileo spacecraft. The energies range from about $5 \mathrm{MeV} /$ nucleon to about 1-2 GeV/nucleon. Four earlier events from solar cycle 21, are taken from Mazur, et al, 1992, and from the Chicago/CRT, and have event integrated fluences from below 1 $\mathrm{MeV} /$ nucleon to $\sim 300 \mathrm{MeV} /$ nucleon. In two cases, this energy range is also extended to beyond 1 $\mathrm{GeV} /$ nucleon. These events are listed in Table 2.

The Analyses

Results

Using the values given in Dr. Sauer's data for $F(>30)$, the event integrated proton fluences $F(>E)$ were calculated using Nymmik's model for kinetic energy in the range $5 \mathrm{MeV}$ to $1000 \mathrm{MeV}$ for each of the 27 events. In referring to specific events, the notation yymmdd is used throughout to identify the event in question. The model curves were then superimposed on the corresponding original data. Because the model contains a size dependent index, it was instructive to examine the aggregate set of proton events in three fluence bins: small: $<10^{7}$, medium: $10^{7}$ to $10^{8}$, and large: $>10^{8}$. With some notable exceptions, it would appear that a flattening of the energy spectra does occur with increasing size, as indicated by the model. In fact, for energies of $30 \mathrm{MeV}$ and greater, reasonable to excellent agreement is attained between data and model for more than half of the events in the set of 27. Figures 1, 2, 3a, and 3b show the kinetic energy spectra of the set of 27 GOES proton events.

Considering first the set of large events, $\left(\mathrm{F}(>30) \geq 10^{8}\right.$ protons $\left./ \mathrm{cm}^{2}\right)$, without even examining the model results, two events stand out as different. Events 891127 and 910323 would appear to have larger spectral indices, i.e. to fall off much more rapidly below $100 \mathrm{MeV}$ than the other seven events in that class. By including the two high energy values, 910323 appears to maintain the steep spectrum, whereas 891127 hardens considerably, thus exhibiting a high energy tail which is unlike any of the other solar proton events examined. It is suggested that this tail is due to galactic cosmic ray protons which were not removed from the data. Comparing the model results with the data for these two events, it clearly does not provide an adequate description of the observed spectra. However, the model predictions for the remaining seven 
events in the class are considerably better, particularly for the huge events 890929 and 891019 . Figures 4 through 7 show the model fits to the data, with the proton spectral index $\gamma+/-1 \sigma$, for events 890929 , 891019,891127 , and 910323 , respectively.

The medium class of events, with $\mathrm{F}(>30)$ integral fluences between $10^{7}$ and $10^{8}$ particles $/ \mathrm{cm}^{2}$ exhibits a slightly different grouping of spectral indices. The class contains a total of just six events, two of which, 910513 and 920625 would appear to have flatter spectra throughout their energy ranges than the other four events. In fact, it would be very difficult to distinguish the values of the spectral indices for these two events from those applying to the large class events. It is interesting to note however, that these two events are the only two in the medium class for which the high energy fluences $F(>355)$ and $F(>685)$ are available. At least partially due to these high energy data, the fits of Nymmik's model appears to be the best for these two events. The remaining four events form a rather tight group, for which the spectra appear to be steeper than the corresponding group of large events, and to be steeper than the model predicts for events of this size. As was seen within the large class events, there appears to be two distinct spectral shapes; one which Nymmik's model describes quite well, and one for which the data fall off much too rapidly at higher energies. Figures 8 and 9, showing events 910513 and 900319 illustrate these two types of event.

As would be expected, the "small" class, for which $F(>30)$ is in the $10^{6}$ range, has the most number of events, twelve in all. Here too, there is an interesting separation into two subclasses. Four of the events, $881213,890520,890725$, and 891115 appear to have much flatter spectra above $10 \mathrm{MeV}$ than even the main group of large events. The model therefore fails to fit any of these four events. Figure 10, event 881213 is representative of this group. Again, it is noted that it is only for these four events that the high energy data is available, and also, that they are not included in the Sauer, 1997 compilation. Two other events in the small class appear to be fit only marginally by the model: 871108 and 890501 . In both cases, the spectrum appears to be too flat at higher energies, but without the data at 355 and $685 \mathrm{MeV}$ it is uncertain whether this is in fact the case. The remaining six events in the class are well described by the model, as is illustrated in figure 11 , event 910825 .

In addition to these 27 events, three of the largest historical events from previous solar cycles were examined. These are: February 23, 1956, November 12, 1960, and August 7, 1972. Details of these events are given in Shea and Smart, 1990, and Wilson, et al, 1997. The spectra are modeled as exponentials in Wilson, et al, 1993. It is seen that the event of August, 1972 falls off very rapidly with increasing energy, and clearly cannot be described by the model, (Figure 12). The February, 1956 event was observed to be very hard at high energies. It is conceivable that this high energy tail is similar to some of the smaller later events, however it still appears to be harder than the model spectral index $\gamma=4.5$, as seen in figure 13 . The event of November, 1960 appears to be the closest of the three to being described by the Nymmik model, (Figure 14).

To summarize the solar proton data analysis, the spectra of 16 of the 30 events analyzed were fit well by the Nymmik model. The remaining 12 events were less well fit, in most cases showing characteristics which could not be explained within the context of the model, and which indicate that two or more classes of spectral shape may be present. It would seem that additional physical parameters are necessary in order to adequately describe the full range of events. It was thought originally that the observed variation in spectral shapes might be due to the position on the solar disk of the causal flare. An examination of available data showed little or no correlation between flare position and spectral characteristics. Several authors, (Reames, et al, 1994; Kahler, et al, 1997), have suggested that temporal variations play a role in the composition and the spectral shape, and have noted that source height variation in the corona may also be of significance. 
Heavy Ions

It has been assumed in the past that the heavy ion component of solar particle events was insignificant so far as radiation dose calculations were concerned. Additionally, very few measurements have been made at satellite altitudes. Early estimates of the composition and spectral characteristics have been done by Mazur, et al, 1992, and Reames, et al, 1994. More recently, Tylka, et al, 1996, 1997; have published some spectral data for oxygen and iron ions for six solar particle events using the IMP-8 spacecraft, as well as several others mentioned above. Following the previous notation, these events are designated: 771122,780428 , $790819,810424,890929$, and 891024 . It will be noted that two of these events correspond to proton events of solar cycle 22 . The event 891024 represents a portion of 891019 .

In order to obtain event integrated fluence spectra for the ion spectra, it is necessary to determine the corresponding proton fluence $F(>30)$. For the four events in solar cycle 21 , this was done using the data in Shea and Smart, 1990. Care had to be taken to align the temporal periods of data collection, as given in the two sources, in order to get the best estimate for the proton fluence corresponding to the heavy ion event. The best illustration of this is found in event 891024 . Examining the original data given in the weekly NOAA Solar Geophysical Data publication, it was seen that event 891019 actually consisted of three pulses of particles. Only the last, (and smallest) pulse coincides with 891024 . Therefore, the value of $F(>30)$ for 891019 had to be corrected. This was done by hand-integrating the temporal flux curve. This integration gave a value of $F(>30)=5.55 \times 10^{8}$ for the period October $24-27$, compared to $4.24 \times 10^{9}$ for the entire event. Figures $15 \mathrm{a}$ and $15 \mathrm{~b}$ show the spectra for oxygen and iron particles respectively, fit with the mean $\gamma^{(z)}$ calculated from the proton fluence $F(>30)$ for this event. A correspondingly good fit was obtained for the September $29^{\text {th }}$, 1989 event.

The model results for the two solar cycle 22 events were very encouraging for both oxygen and iron spectra. In the published data for these events there is almost no indication of a flattening below 30 $\mathrm{MeV} /$ nucleon. Consequently, we ran the model without the droop calculation for energies below this energy. It is observed that the fits are excellent. One possible improvement in the model might be to perform the droop calculation with an expression eliminating the A/Q dependence, which seemed to drive the low energy portion of the spectrum far too low.

The model results for the other four events were not quite as good. For 771122 , the oxygen model paralleled the data about an order of magnitude too high, until intersecting what are possibly galactic cosmic ray (GCR) values at approximately $200 \mathrm{MeV} /$ nucleon. The iron spectrum appeared to match the data quite reasonably above $\sim 20 \mathrm{MeV} /$ nucleon. This would indicate that the ratio of $\mathrm{O} / \mathrm{Fe}$ is not correct, or varies from event to event as indicated in the references. The oxygen data for event 780428 fell off more rapidly with increasing energy than the model predicts, even appearing to be somewhat like the exponentialtype proton spectra. The corresponding iron spectrum has a similar form, although not so extreme. The model calculation presents a barely acceptable fit to the data. Both the August 1979 (790819), and the April, 1981 (810424) events presented good model fits for both oxygen and iron data.

To summarize the heavy ion model results, very good fits were obtained in four out of the six cases. The model iron results appeared more robust in the remaining cases. It should be noted again that no droop calculations were used in the heavy ion spectra model; and that the results are quite sensitive to the values taken for the event integral proton fluences. In some cases there also appears to be uncertainty concerning whether the data represent GCR or solar particles, particularly at energies above $\sim 200 \mathrm{MeV} /$ nucleon. This could indicate that the solar heavy ions do indeed represent a more serious radiation dose hazard to astronauts than has been assumed previously, particularly in cases such as the October 1989 event, in which possibly only part of the dose due to heavy ions was observed. This needs to be examined carefully in the near future. 


\section{References:}

Feynman, J., Spitale, G., and Wang, J.; J. Geophys. Research, 98, No. A8, 13281-13294, 1993

Kahler, S. W., Smart, D. F., and Sauer, H.; XXVth ICRC, South Africa, August, 1997

Mazur, J. E., Mason, G. M., Klecker, B., and McGuire, R. E.; Ap.J. 401, 398-410, 1992

Nymmik, R. A., International Standard ISO WD 15391, 1997

Reames, D. V., Meyer, J. P., and von Rosenvinge, T. T.; Ap J. Suppl. 90:649-667, 1994

Sauer, H. H. ; XXIII ICRC, Calgary, V3. 250-253, 1993

Sauer, H. H. private communication: "Preliminary Solar Cycle 22 EventFluences, (1995)", 1997

Shea, M. A., and Smart, D.F. Solar Physics 127, 297-320, 1990

Tylka, A. J., Dietrich, W. F., Boberg, P. R., Smith, E. C., and J. H. Adams, Jr. ; IEEE Transactions on Nuclear Science, 43, No. 6, 2758-2766, December, 1996

Tylka, A. J., Dietrich, W. F., and Boberg, P. R.; XXV ICRC, South Africa, August, 1997

Wilson, J. W. , Nealy, J. E., and Schimmerling, W., NASA Technical Memorandum 4422, 1993

Wilson, J. W., Cucinotta, F. A., Jones, T. D., and Chang, C. K., NASA Technical Paper 3643, April, 1997

Table 1 Proton Events

\begin{tabular}{|c|c|c|c|}
\hline $\begin{array}{l}\text { Event ID } \\
\text { (yymmdd) }\end{array}$ & Onset Date & $\begin{array}{l}\text { Integral Fluence, } \mathrm{F}(>30) \\
\left.\text { (protons } / \mathrm{cm}^{2}\right)\end{array}$ & Solar Disk Position of Flare \\
\hline 560223 & February 23, 1956 & $1.0 \times 10^{9}$ & \\
\hline 601112 & November 12,1960 & $9.72 \times 10^{9}$ & \\
\hline 720804 & August 4,1972 & $5.0 \times 10^{9}$ & \\
\hline 871108 & November 8,1987 & $1.20 \times 10^{6}$ & $\mathrm{~N} 31 \mathrm{~W}>90$ \\
\hline 881213 & December 13,1988 & $8.52 \times 10^{6}$ & N27 E37 \\
\hline 890308 & March 8, 1989 & $4.91 \times 10^{7}$ & N35 E46 \\
\hline 890411 & April 11, 1989 & $4.69 \times 10^{6}$ & N37 W12 \\
\hline 890501 & May 1,1989 & $1.28 \times 10^{6}$ & S18 Wo \\
\hline 890520 & May 20,1989 & $5.96 \times 10^{6}$ & S20 E5 \\
\hline 890725 & July 25,1989 & $8.11 \times 10^{6}$ & N28 W84 \\
\hline 890812 & August 12,1989 & $1.52 \times 10^{9}$ & S18 W36 \\
\hline 890929 & September 29, 1989 & $1.39 \times 10^{9}$ & S25 $\mathrm{W}>90$ \\
\hline 891019 & October 19,1989 & $4.24 \times 10^{9}$ & S27 E10 \\
\hline 891115 & November 15,1989 & $5.70 \times 10^{6}$ & N11 W33 \\
\hline
\end{tabular}




$\begin{array}{llll}891127 & \text { November 27, 1989 } & 1.31 \times 10^{8} & \text { N25 W3 } \\ 900319 & \text { March 19, 1990 } & 2.17 \times 10^{7} & \text { N33 W43 } \\ 900428 & \text { April 28, 1990 } & 4.80 \times 10^{6} & \text { unknown } \\ 900521 & \text { May 21, 1990 } & 1.38 \times 10^{8} & \text { N35 W36 } \\ 900801 & \text { August 1, 1990 } & 7.41 \times 10^{6} & \text { N20 E45 } \\ 910131 & \text { January 31, 1991 } & 1.28 \times 10^{6} & \text { S17 W35 } \\ 910323 & \text { March 23, 1991 } & 1.78 \times 10^{9} & \text { S26 E28 } \\ 910513 & \text { May 13,1991 } & 1.82 \times 10^{7} & \text { S10 W }>0 \\ 910604 & \text { June 4, 1991 } & 7.85 \times 10^{8} & \text { N30 E70 } \\ 910611 & \text { June 11, 1991 } & 6.27 \times 10^{8} & \text { N31 W20 } \\ 910629 & \text { June 29,1991 } & 3.00 \times 10^{7} & \text { N30 E >90 } \\ 910825 & \text { August 25, 1991 } & 3.41 \times 10^{6} & \text { N24 E77 } \\ 920509 & \text { May 9, 1992 } & 1.32 \times 10^{7} & \text { S26 E8 } \\ 920625 & \text { June 25, 1992 } & 4.77 \times 10^{7} & \text { N9 W67 } \\ 921102 & \text { November 2,1992 } & 2.64 \times 10^{8} & \text { S25 W >90 } \\ 940220 & \text { February 20, 1994 } & 4.13 \times 10^{6} & \text { N11 W14 }\end{array}$

Table 2 Heavy Ion Events

$\begin{array}{clc}\begin{array}{c}\text { Event ID } \\ \text { (yymmdd) }\end{array} & \text { Onset Date } & \begin{array}{c}\text { Proton Integral Fluence } F(>30) \\ \text { (protons } / \mathrm{cm}^{2} \text { ) }\end{array} \\ 771122 & \text { November 22, 1977 } & 6.3 \times 10^{7} \\ 780428 & \text { April 28, 1978 } & 2.47 \times 10^{8} \\ 790819 & \text { August 19, 1979 } & 9.5 \times 10^{7} \\ 810424 & \text { April 24, 1981 } & 7.0 \times 10^{7} \\ 890929 & \text { September 29.1989 } & 1.39 \times 10^{9} \\ 891024 & \text { October 24, 1989 } & 5.55 \times 10^{8}\end{array}$




\section{Figure Captions}

Figure 1: "Large" solar proton events, with integrated event fluences $\mathrm{F}(>30 \mathrm{MeV}) \geq 10^{8}$ particles $/ \mathrm{cm}^{2}$.

Figure 2: "Medium" events, with integrated event fluences $10^{7} \leq \mathrm{F}(>30)<10^{8}$ particles $/ \mathrm{cm}^{2}$.

Figures 3a, 3b: "Small" events, with $10^{6} \leq \mathrm{F}(>30)<10^{7}$ particles $/ \mathrm{cm}^{2}$. The class is divided into two in order to show the individual events more clearly.

Figures 4 through 11: Representative individual events (GOES data), from solar cycle 22, together with Nymmik's model spectra. Also shown are the $+/-1 \sigma_{\gamma}$ values for the calculated $\gamma$.

Figure 12: The August 4, 1972 event. Wilson, et al, (1997), describe the available data for the differential fluence, (protons $/ \mathrm{cm}^{2}-\mathrm{MeV}$ ), by the expression: $\Phi(\mathrm{E})=3.0 \times 10^{8} \exp [-(\mathrm{E}-30) / 26.5]$. This expression is integrated, and plotted together with Feynman, et al's (1993) data, as is the Nymmik model spectrum, with $+/-1 \sigma$.

Figure 13: The February 1956 event. The event data are summarized by Wilson, et al (1997) with the expression: $\Phi(E)=6.0 \times 10^{7} \exp [-(E-10) / 25]+9.4 \times 10^{5} \exp [-(E-100) / 320]$ protons $/ \mathrm{cm}^{2}-\mathrm{MeV}$. This expression is integrated, and compared to the Nymmik model spectrum for the fluence $F(>30 \mathrm{MeV})$, which is also shown.

Figure 14: The November 1960 event. Wilson, et al, (1997) summarize these data with the expression: $\Phi(E)=6.3 \times 10^{8} \exp [-(E-10) / 12]+4.9 \times 10^{6} \exp [-(E-100) / 80]$. This expression is integrated and plotted, together with the corresponding spectrum from Nymmik's model.

Figures 15a and 15b: These plots show the Tylka, et al data for the oxygen and iron particles for the October 24, 1989 event. Nymmik's model spectra for these species are calculated from the corresponding proton fluence $F(>30 \mathrm{MeV})$, for the period 24-27 October. 
Integral Fluence (protons/cm2)

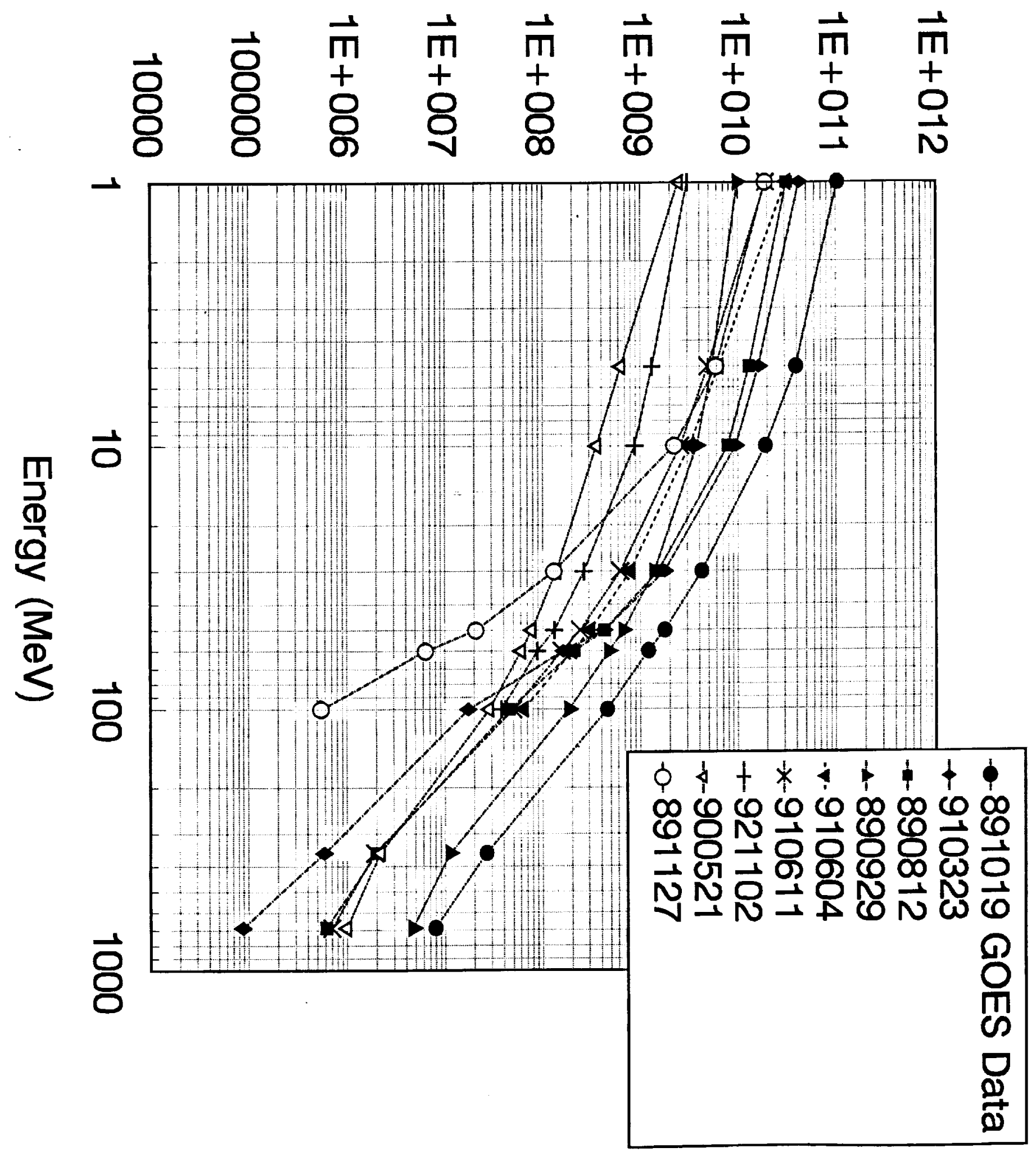




\section{Integral Fluence (Protons/cm2)}

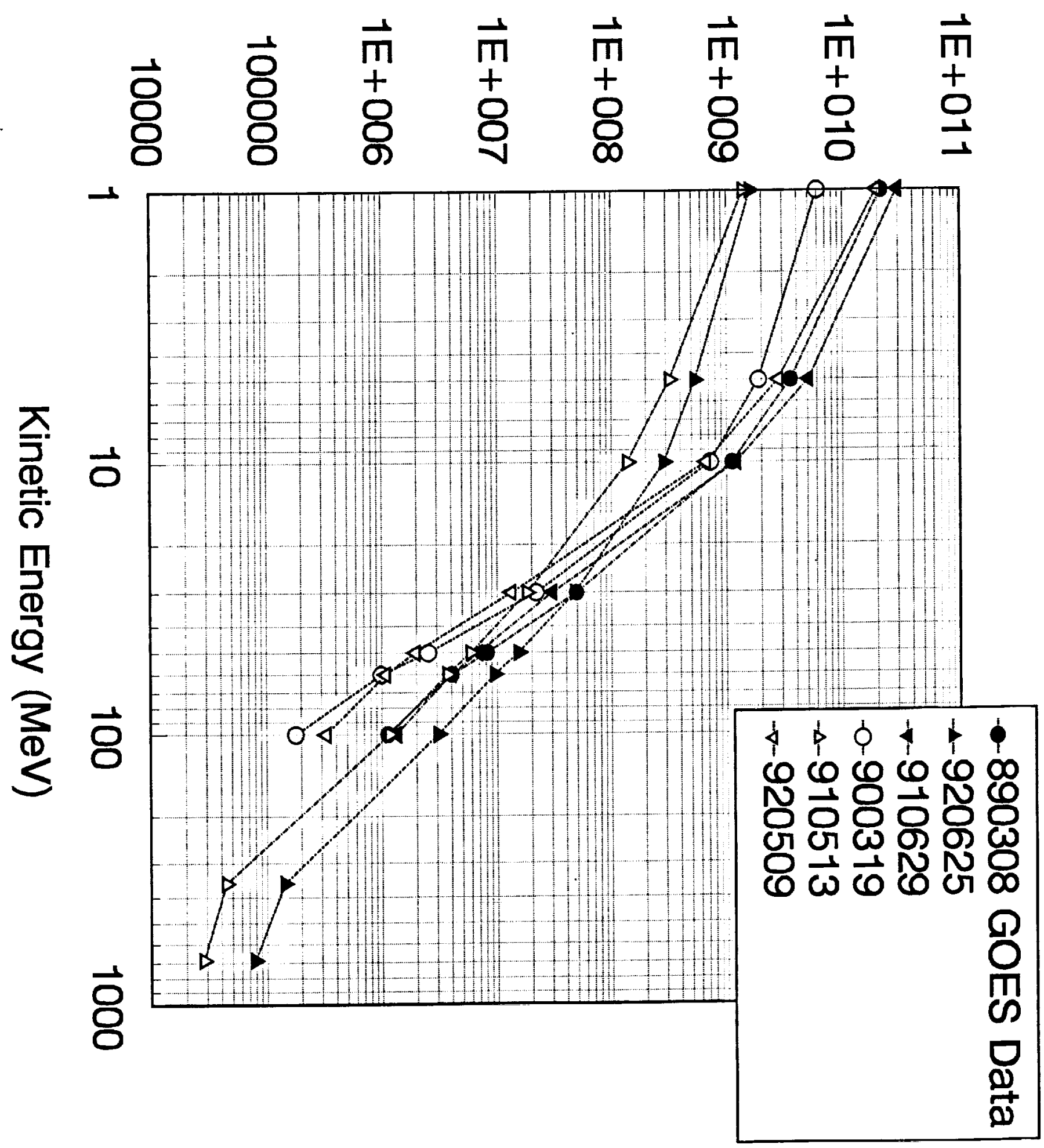




\section{Integral Fluence (Protons/cm2)}

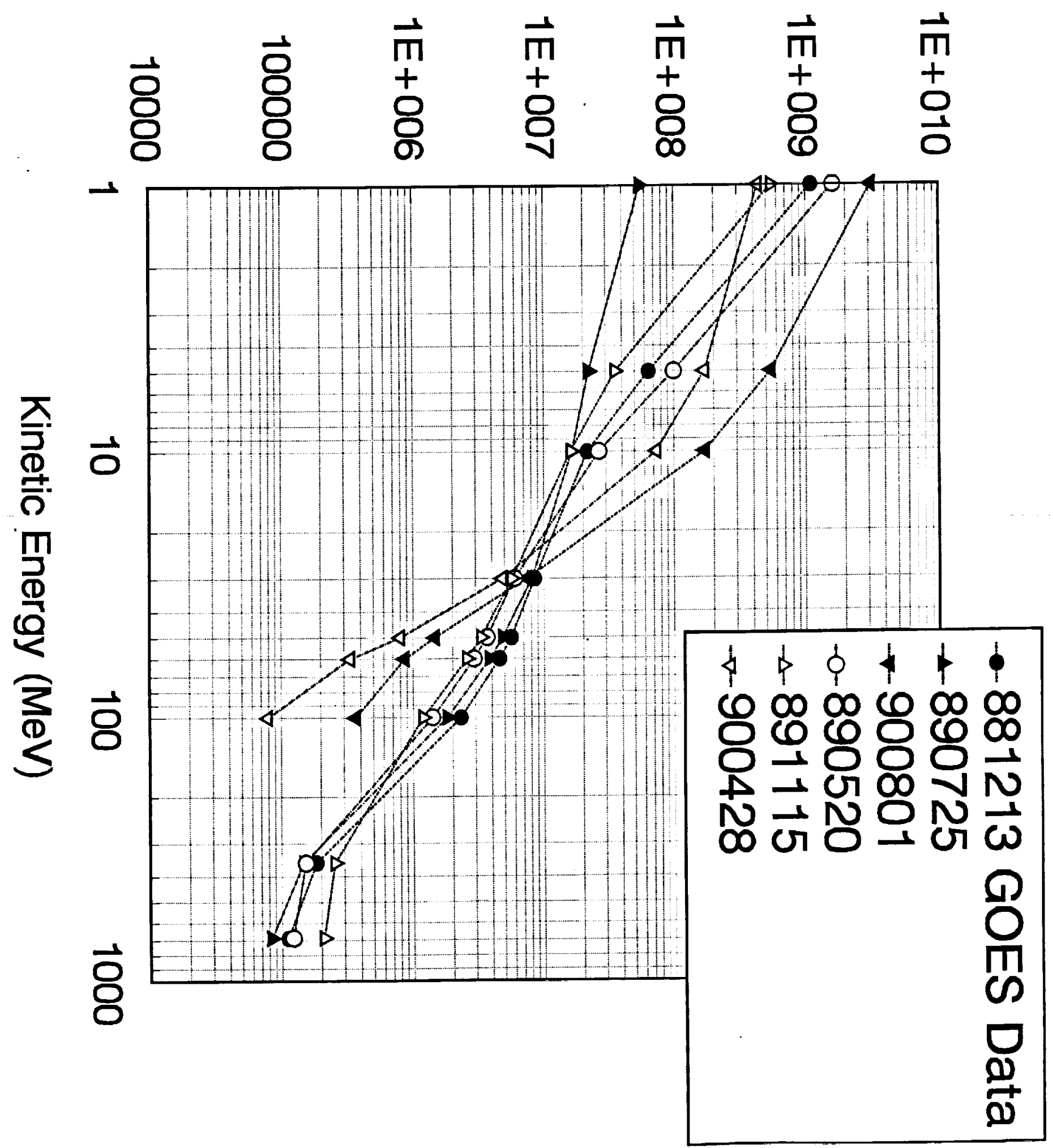




\section{Integral Fluence (protons/cm2)}

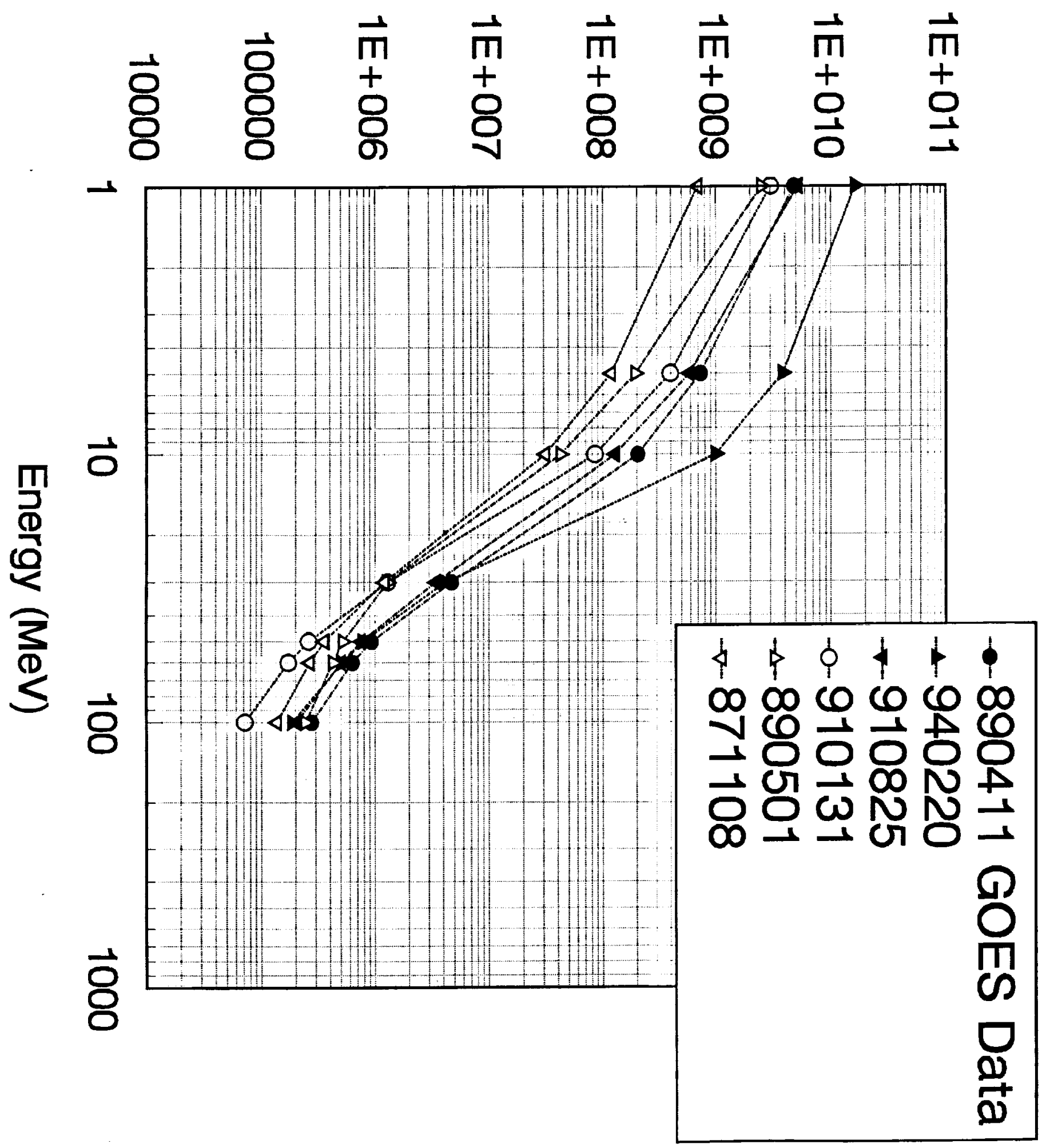




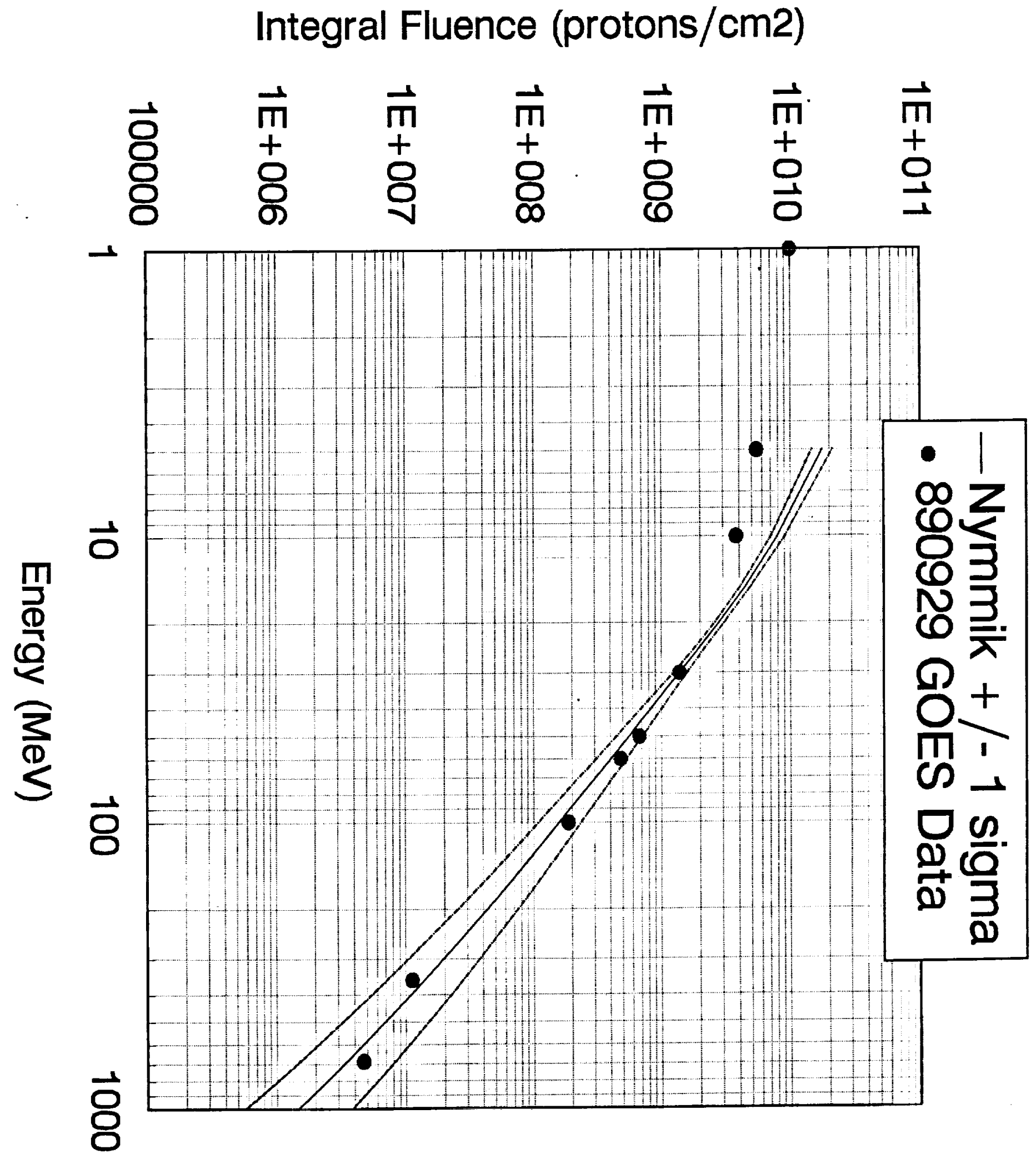




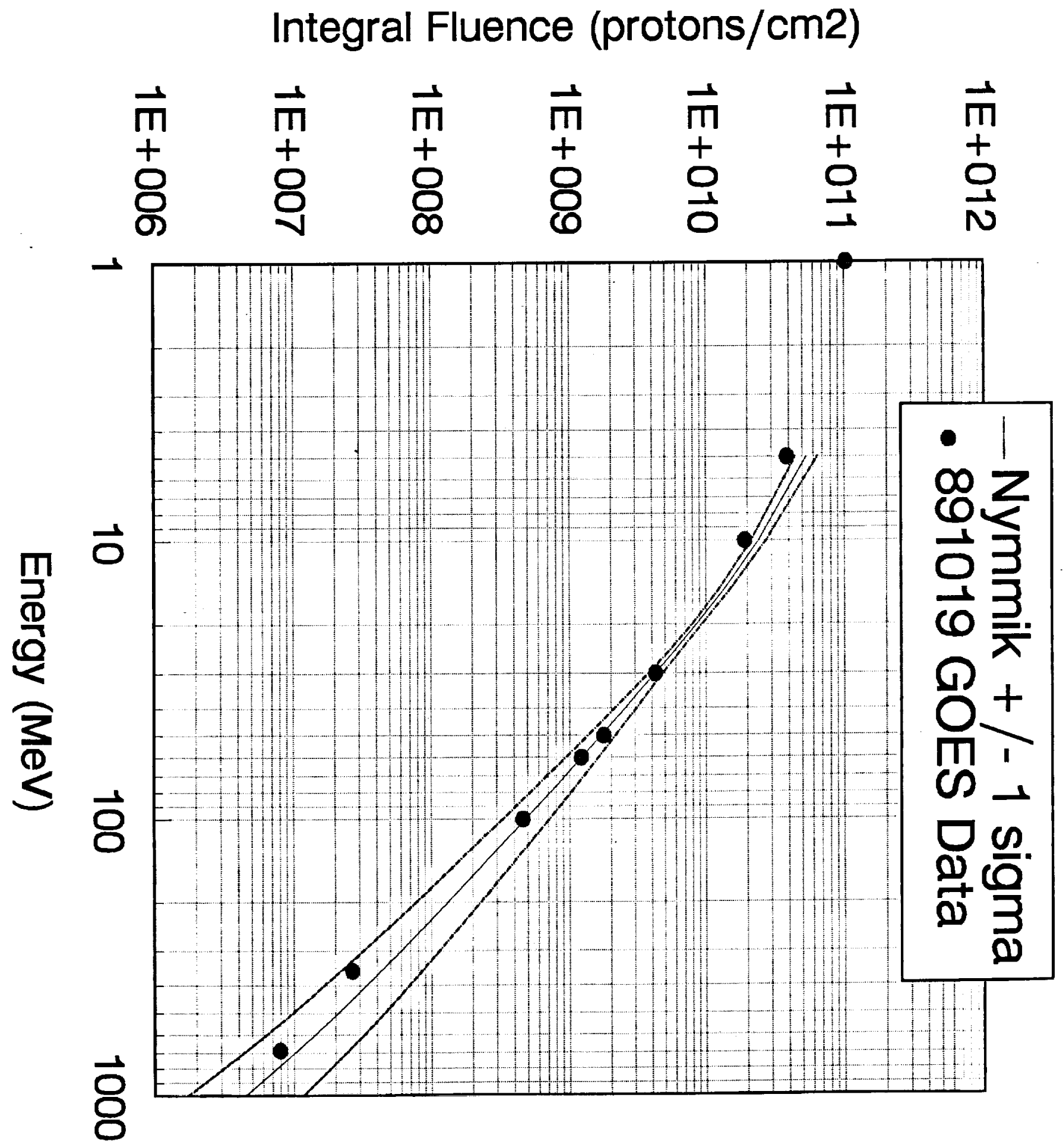




\section{Integral Fluence (protons/cm2)}

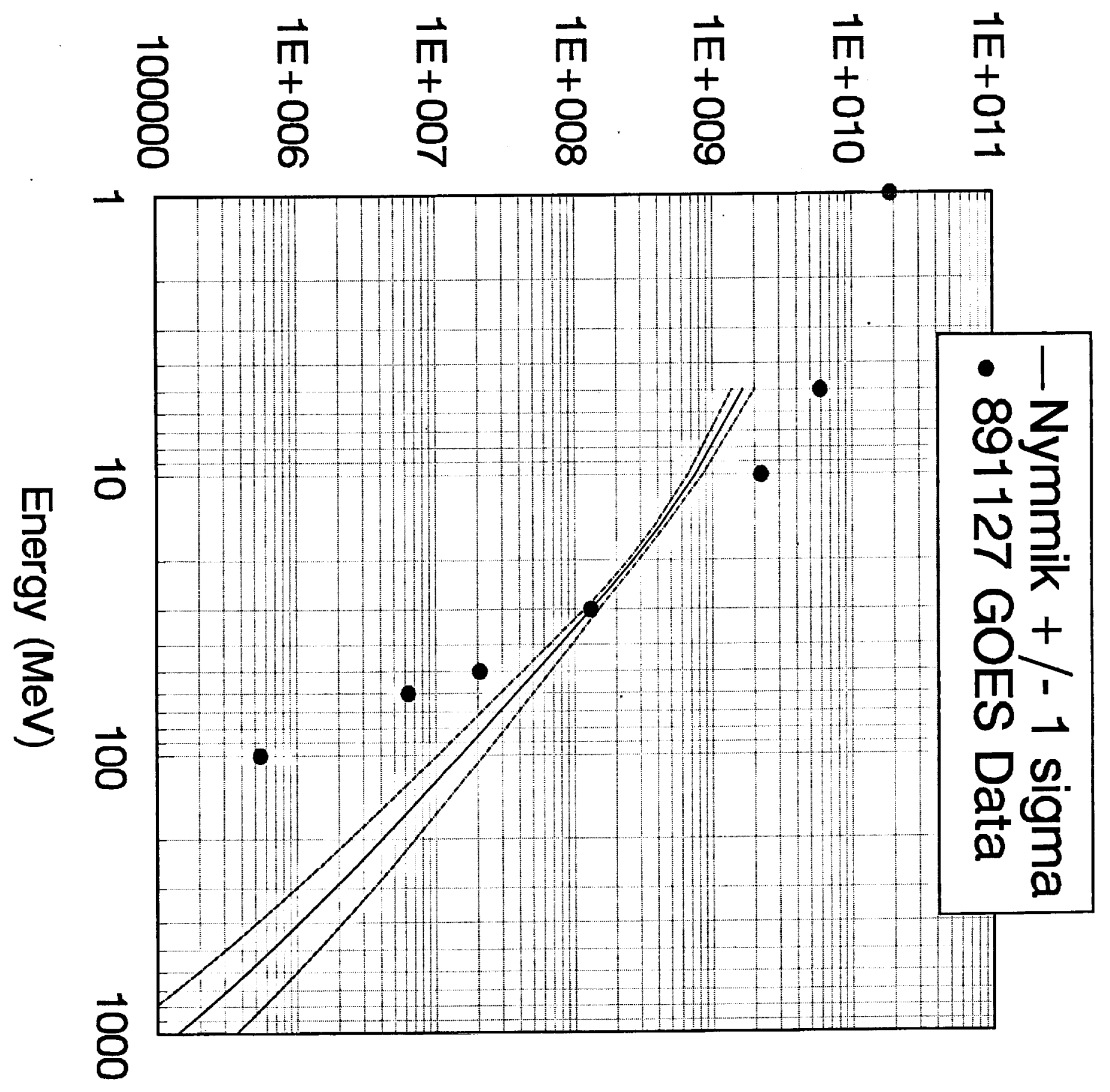




\section{Integral Fluence (protons/cm2)}

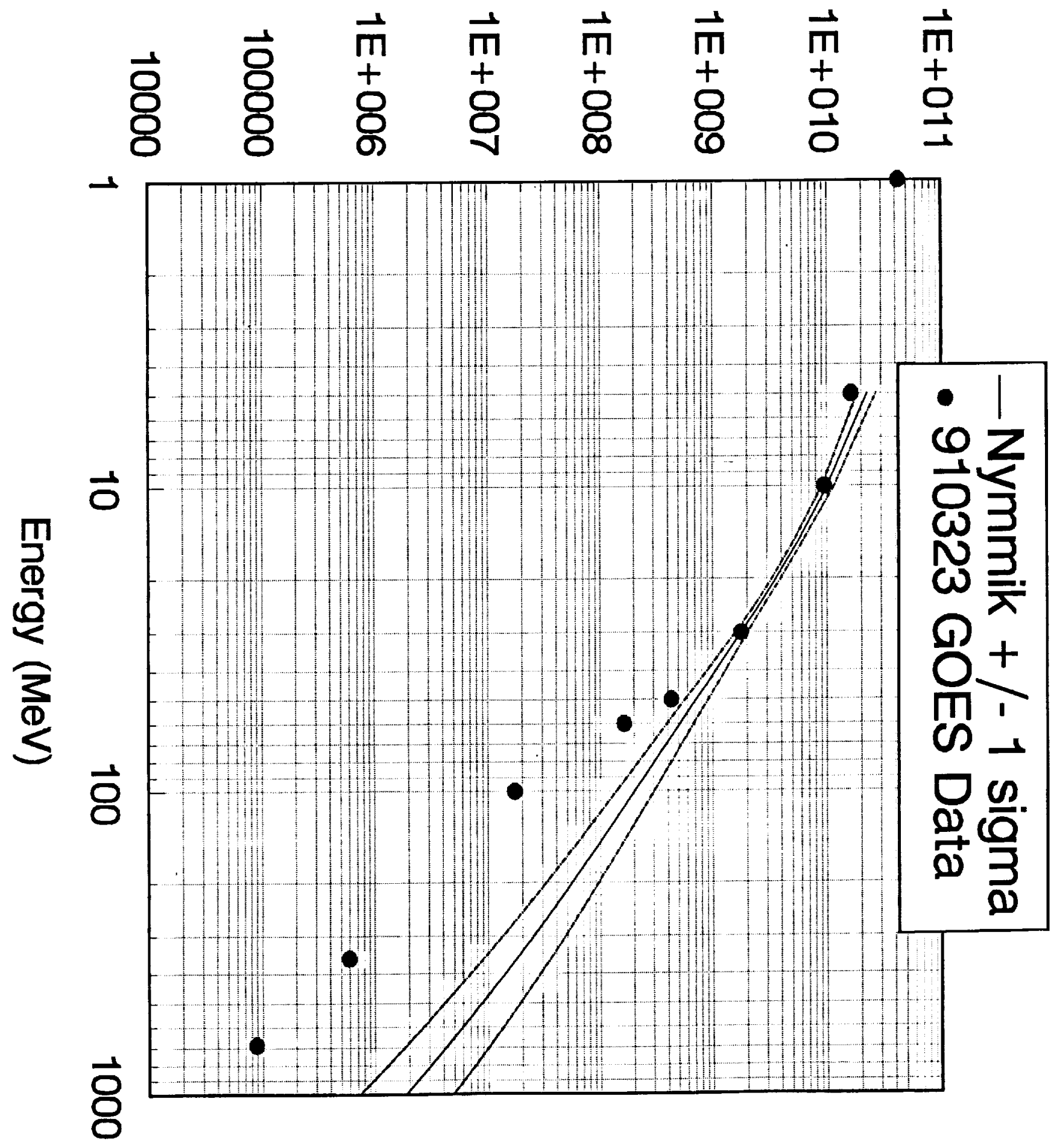


Integral Fluence (protons/cm2)

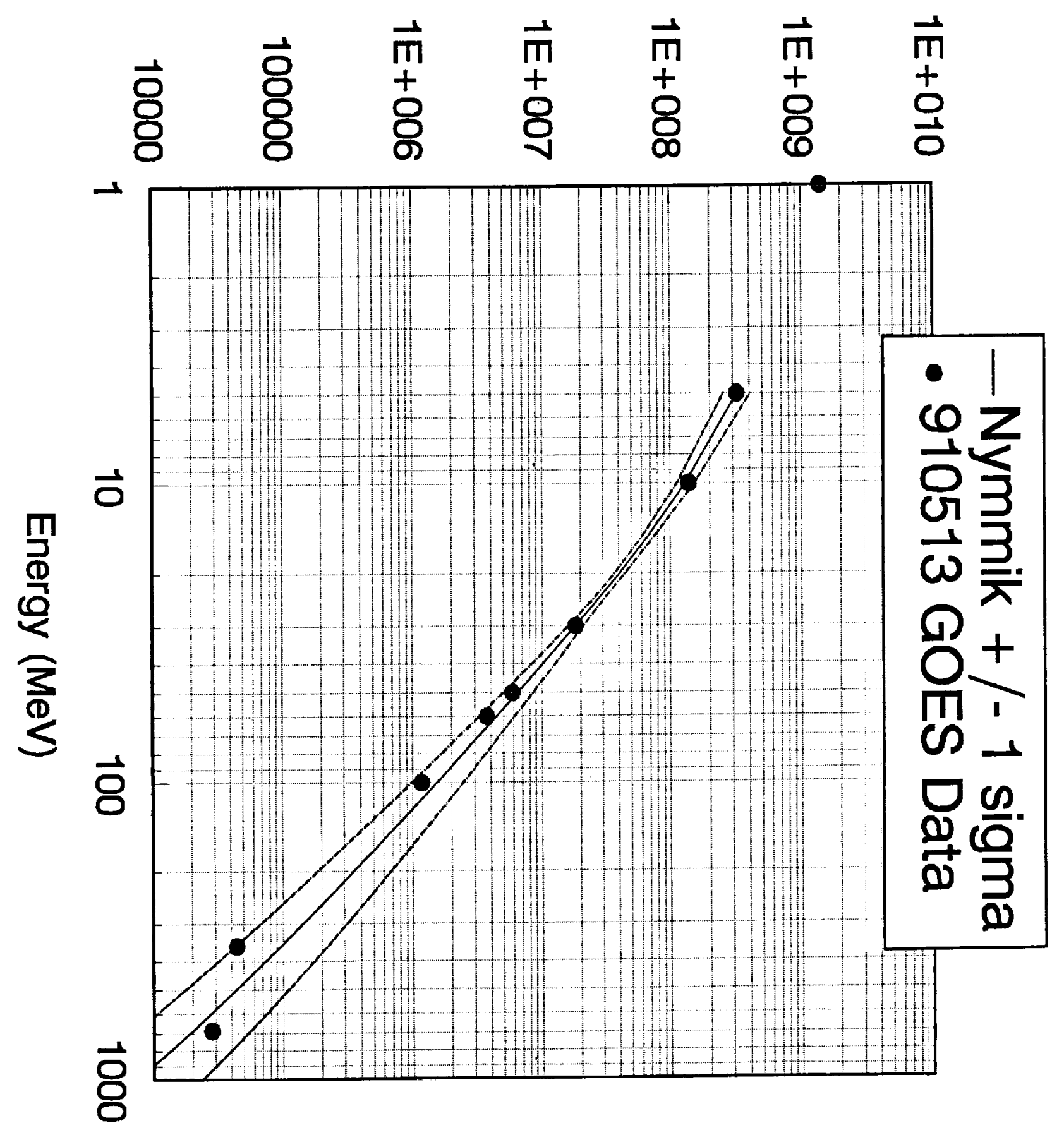




\section{Integral Fluence (Protons/cm2)}

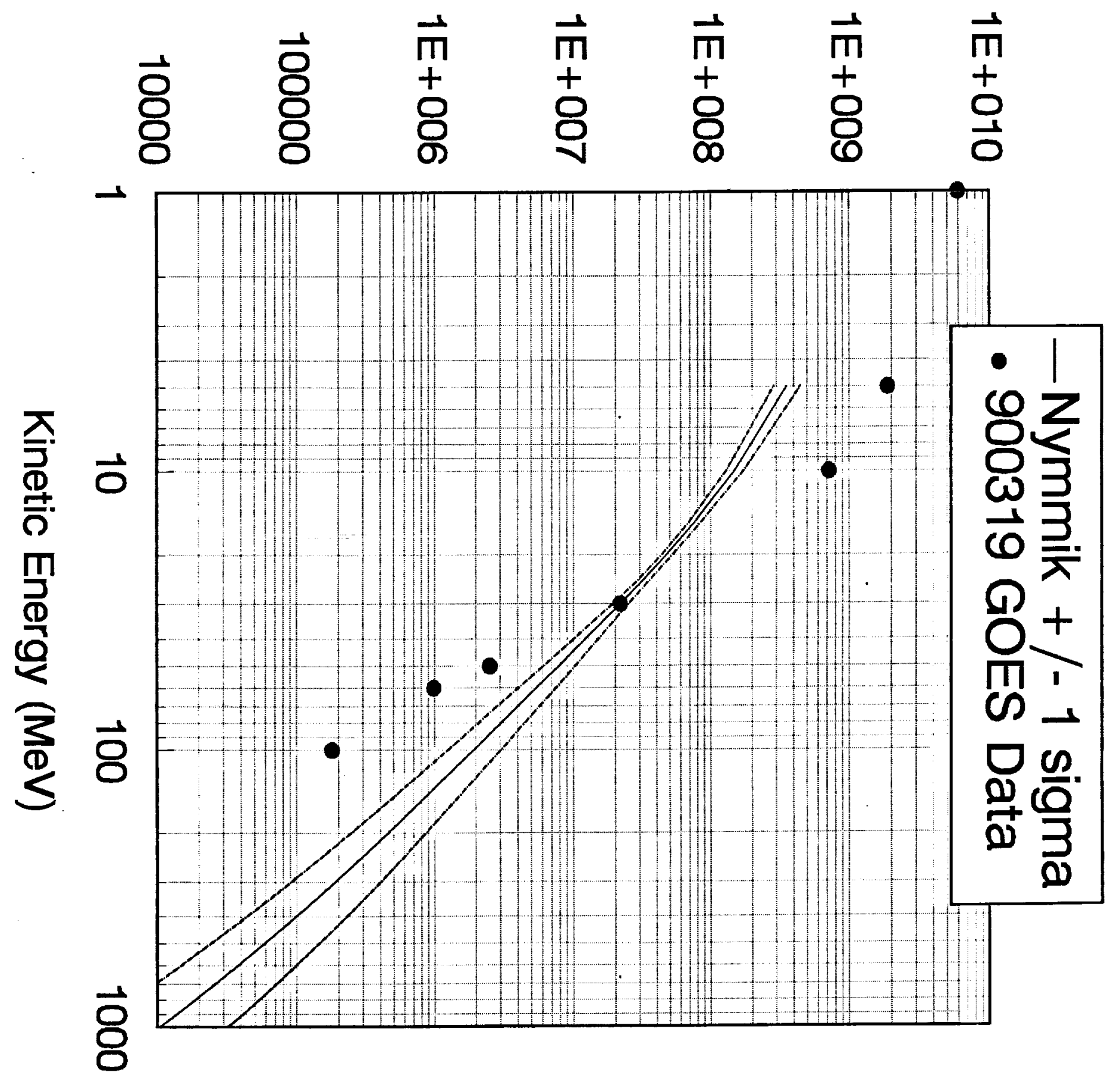




\section{Integral Fluence (Protons/cm2)}

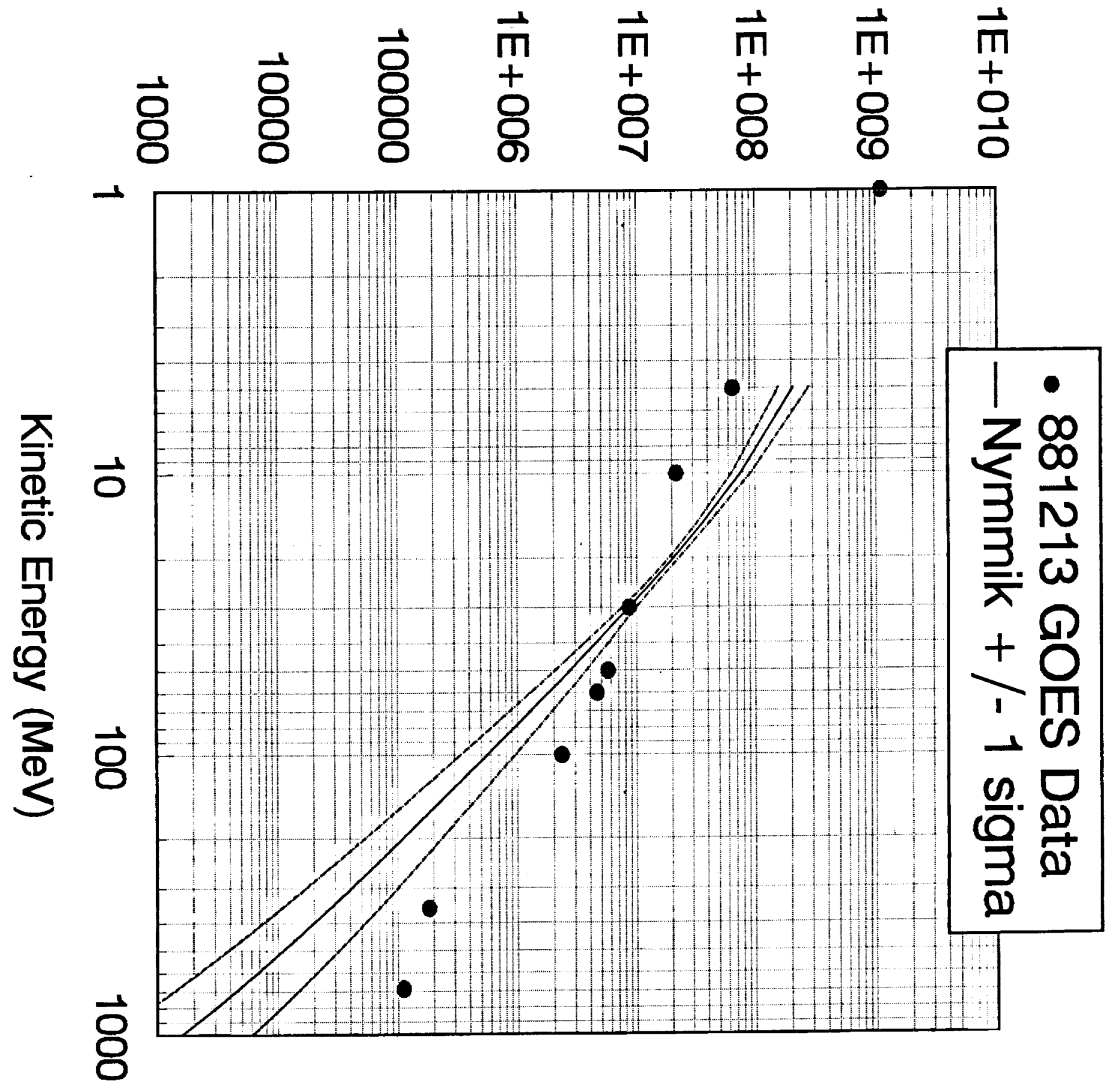


- Integral Fluence (Protons/cm2)

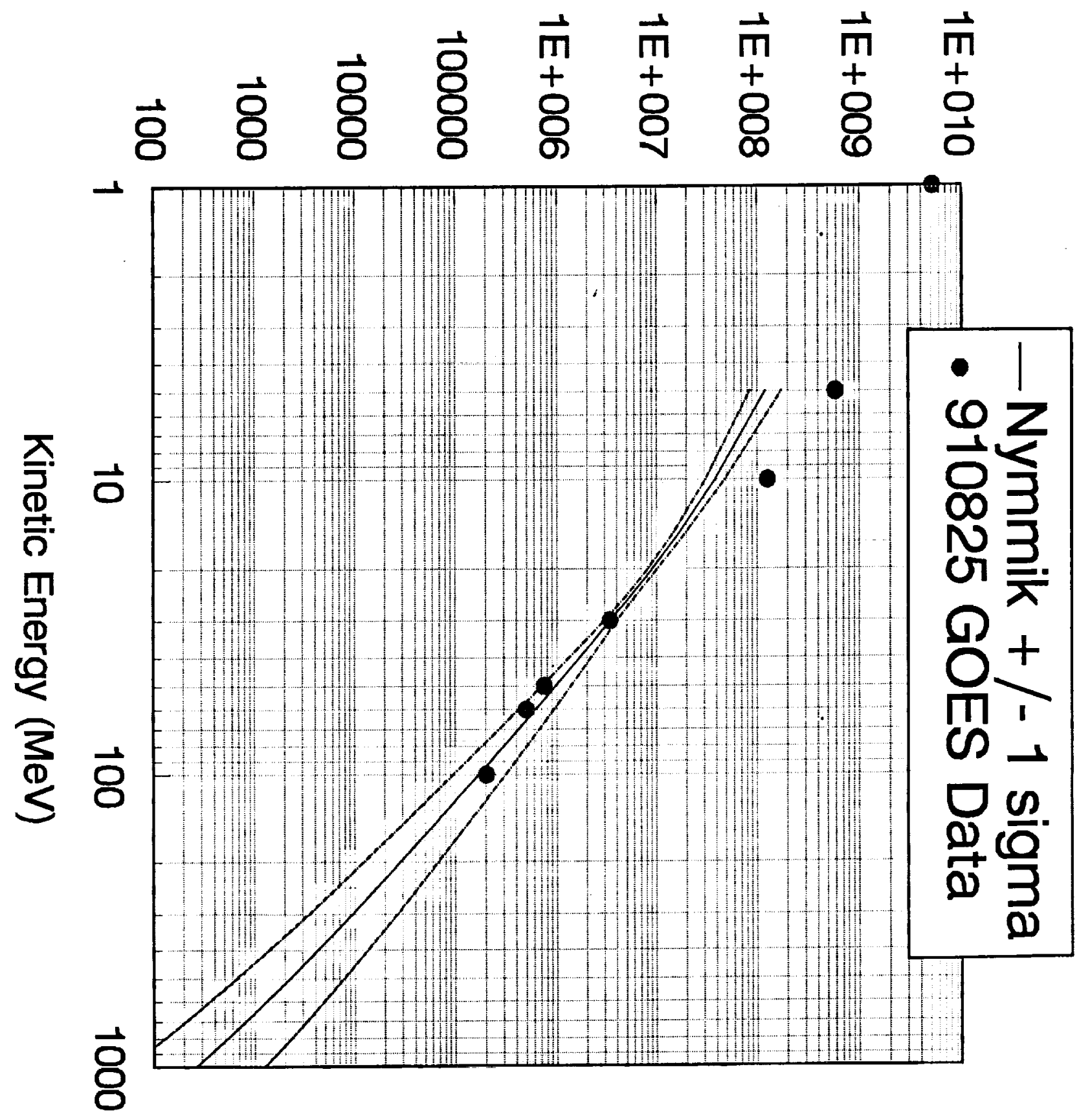


Integral Fluence (particles/cm2)

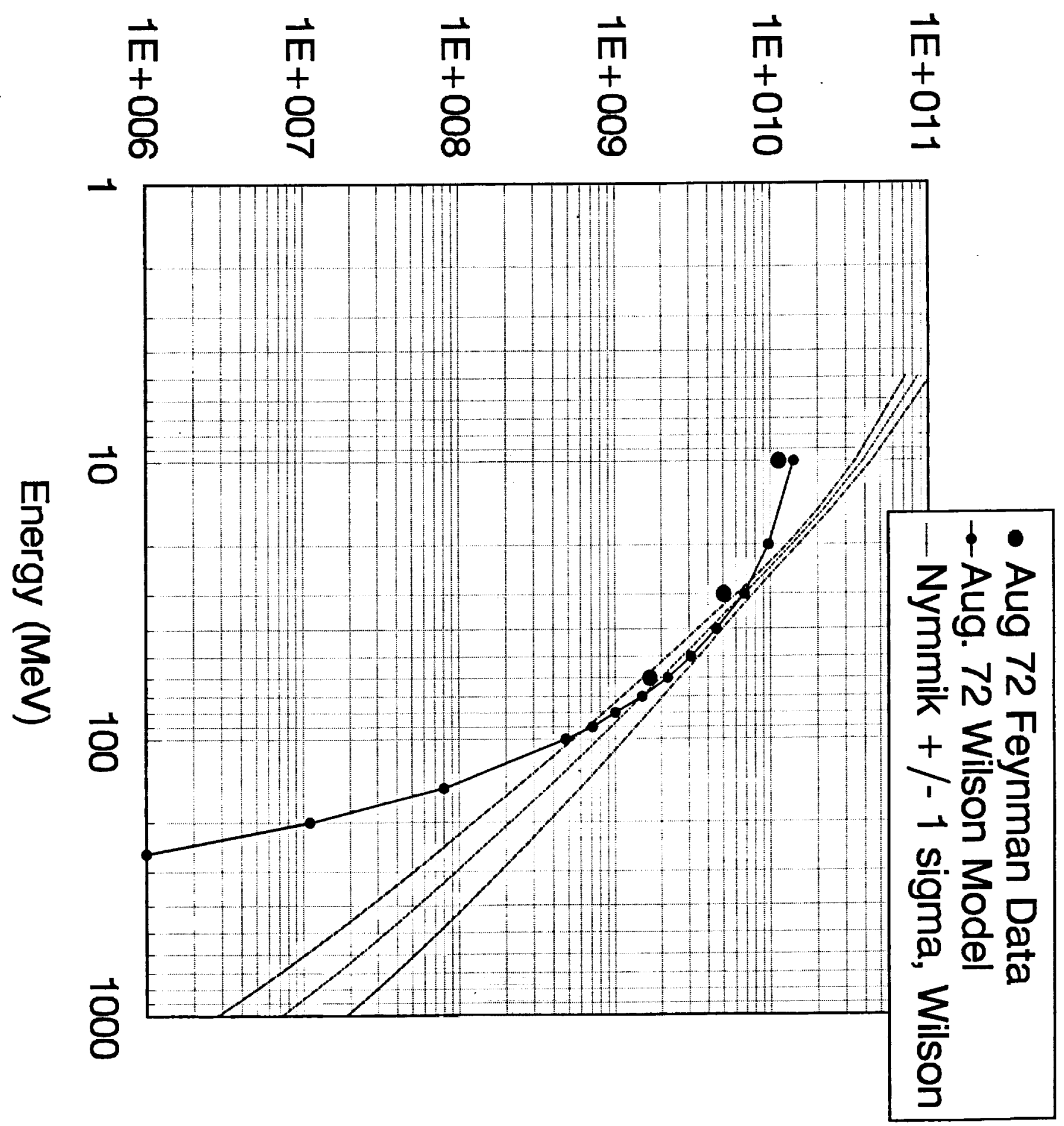


Integral Fluence (particles/cm2)

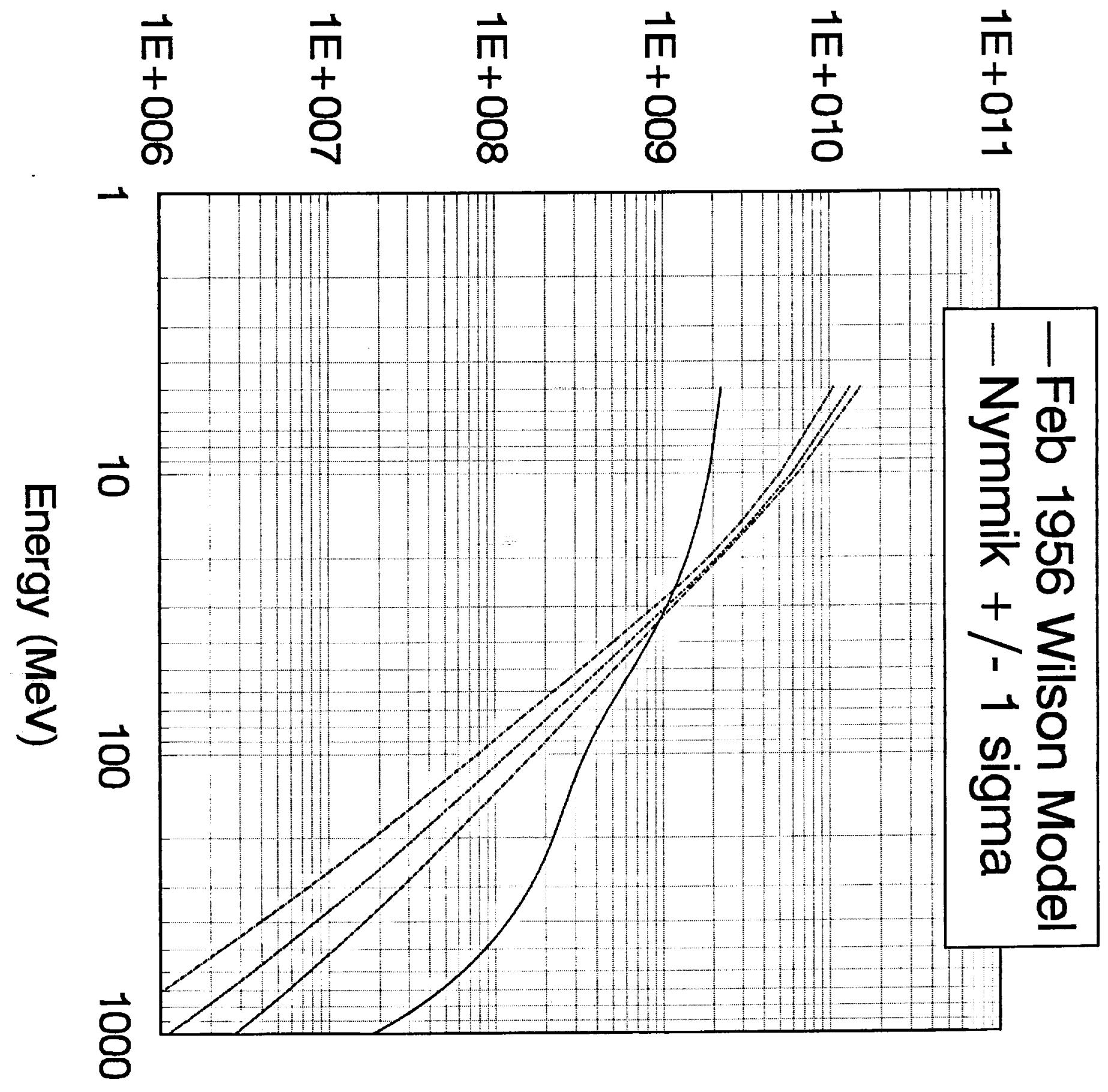


Integral Fluence (particles/cm2)

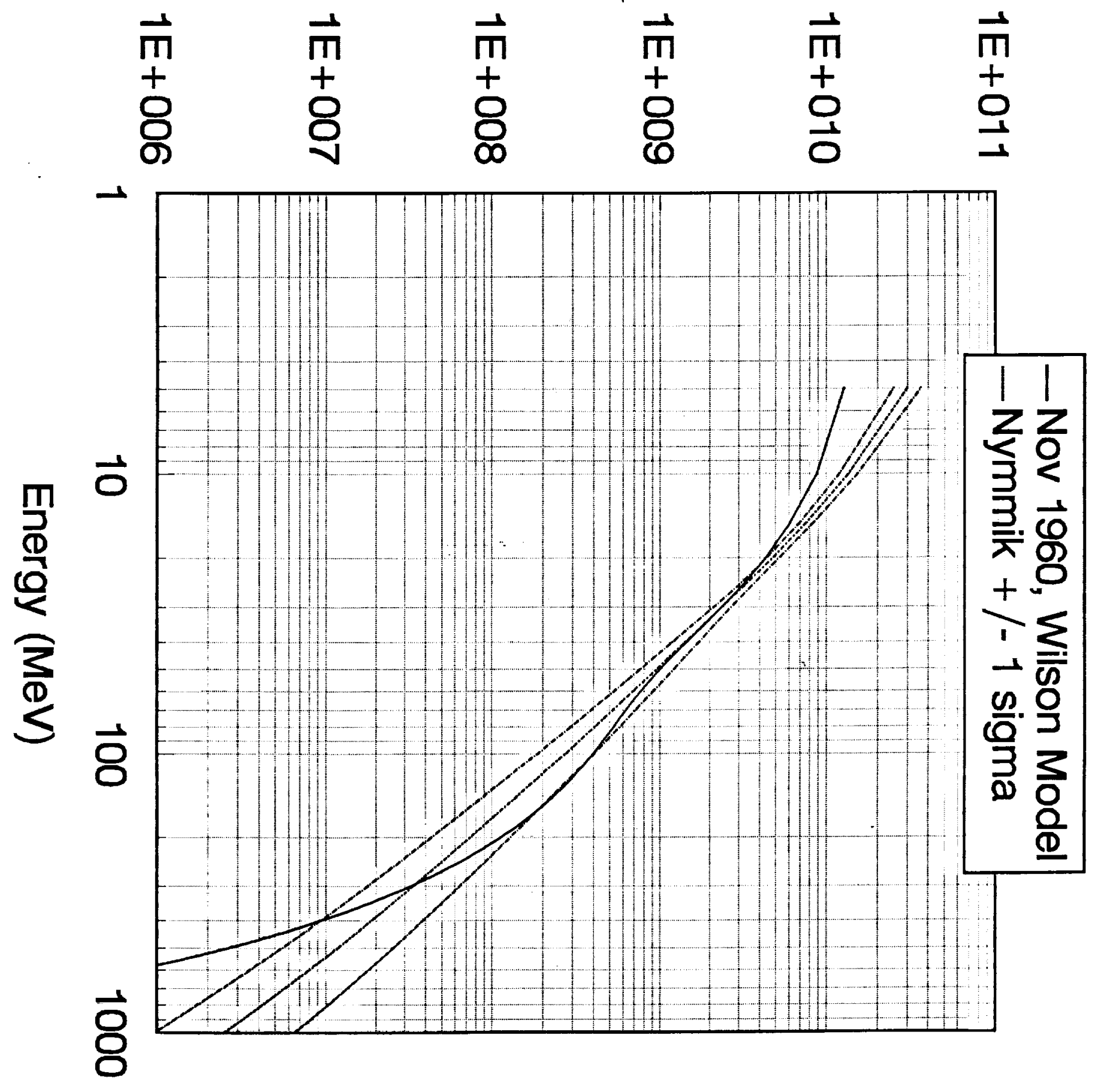



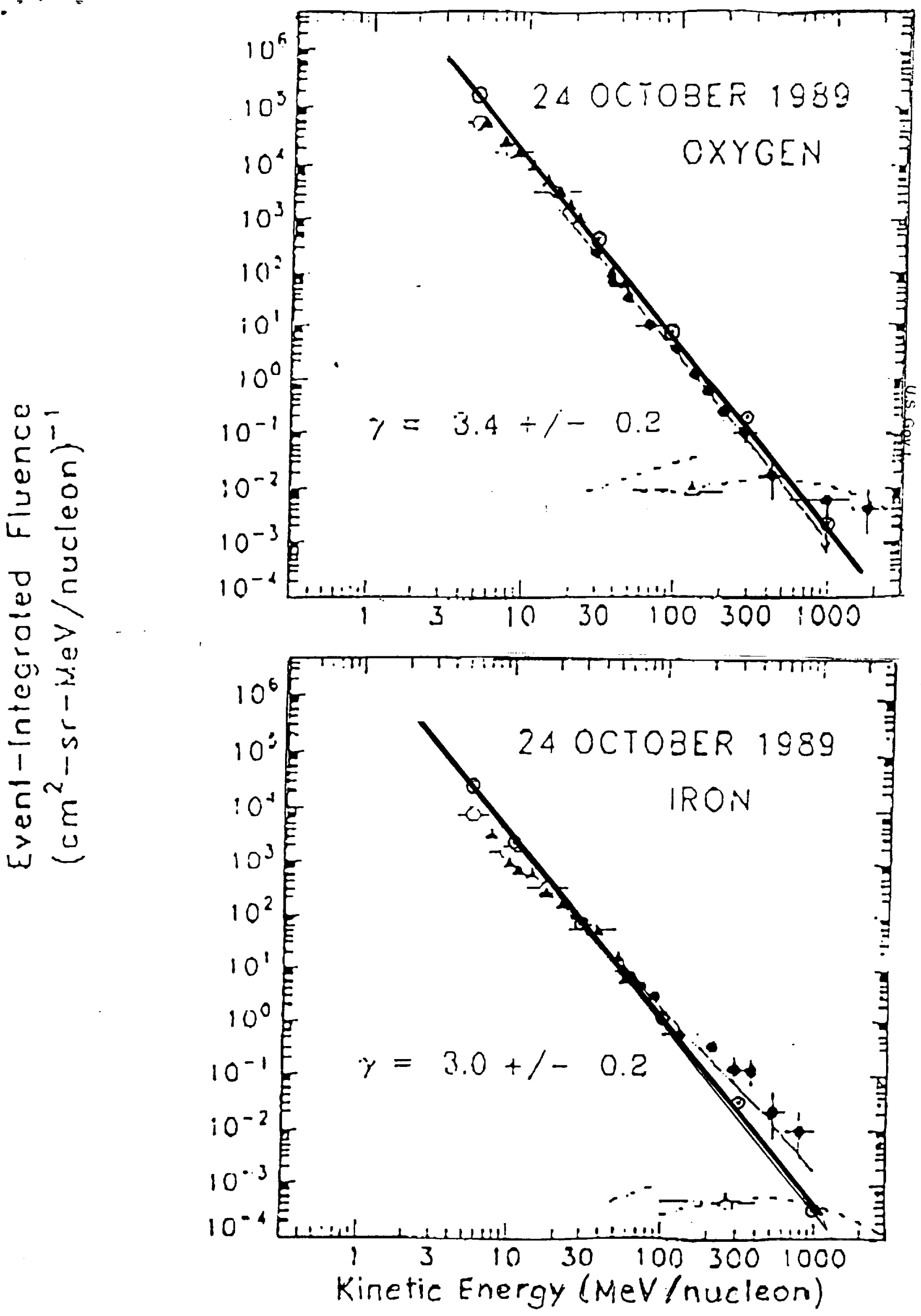